\title{
Respiratory Mechanics in Infants and Young Children Before and After Repair of Left-to-Right Shunts ${ }^{1}$
}

\author{
EUGENIO BARALDI, MARCO FILIPPONE, ORNELLA MILANESI, GIAMPIERO MAGAGNIN, \\ FEDERICA VENCATO, PATRIZIA BARBIERI, P. ANDREA PELLEGRINO. AND \\ FRANCO ZACCHELLO \\ Department of Pediatrics, University of Padova School of Medicine, Padera, Italy
}

\begin{abstract}
In an attempt to investigate the relationship between respiratory mechanics and pulmonary hemodynamics, we evaluated pulmonary function in 31 infants with left-to-right shunts and subsequent high pulmonary blood flow, undergoing cardiac surgery. Measurements were performed $1 \mathrm{~d}$ before and repeated $10 \mathrm{~d}$ and 4-5 wh after correction. The age of the patients ranged from $4 \mathrm{~d}$ to 24 mo, body weight from 2.7 to $11.8 \mathrm{~kg}$. Pulmonary artery pressure, assessed by Doppler echocardiography, was preoperatively elevated in 23 patients (group 1), whereas it was within normal values in eight infants (group 2). Respiratory mechanics were measured using the single-breath occlusion technique in sedated infants. To evaluate specific compliance, functional residual capacity was determined by using an open circuit nitrogen washout technique. $A$ reduced preoperative compliance value (mean with $95 \%$ confidence interval) was found in group 1: 34.8 (26.5-43.1) $\mathrm{mL} \cdot \mathrm{kPa}^{-1}$. After hemodynamic correction, a progressive significant $(p<0.01)$ improvement was demonstrated at $10 \mathrm{~d}$ and 1 mo with values of $47.5(39.2-55.8) \mathrm{mL} \cdot \mathrm{kPa}^{-1}$ and $56.5(45.6-67.4) \mathrm{mL} \cdot \mathrm{kPa}^{-1}$, respectively. A similar trend was noted evaluating specific compliance with values of $0.27(0.24-0.30) \mathrm{kPa}^{-1}$ and $0.44(0.42-0.46) \mathrm{kPa}^{-1}$, respectively before and after surgery. Preoperative functional residual capacity value was $130(100-160) \mathrm{mL}$. In group 2, normal preoperative compliance values were obtained, without significant changes after surgery. In both groups, resistance was within the normal range both before and after surgical correction, and functional residual capacity did not change either. No correlations were found between compliance and pulmonary artery pressure and pulmonary blood flow values. In conclusion, these results show that infants with left-to-right shunts and pulmonary hypertension present with reduced compliance of the respiratory system that rapidly improves after cardiac surgery. (Pediatr Res 34: 329-333, 1993)
\end{abstract}

\section{Abbreviations}

CIID, congenital heart disease

PBF, pulmonary blood flow

PAP, pulmonary artery pressure

Crs, compliance of the respiratory system

Rrs, resistance of the respiratory system

FRC, functional residual capacity

SCrs, specific compliance of the respiratory system

Received September 22, 1992; accepted March 25, 1993.

Correspondence: Dr. Eugenio Baraldi, Department of Pediatrics, University of Padova, Via Giustiniani 3,35128 Padova-Italy.

' Presented at the 1992 International Conference of the American Thoracic Society, Miami, FL.
Rpa/Ao, right pulmonary artery/aorta

$\mathrm{CV}$, coefficient of variation

$\mathrm{CI}$, confidence interval

The heart and the lung are considered an inseparable structure whose functions are closely coupled and whose interrelationship is complex and influenced by many factors. Because the critical, functional, and anatomical linking structure of the unit is the pulmonary vascular tree, this represents the way by which disorders of one component can affect the other. Infants with leftto-right shunts offer a pattern of respiratory mechanics impairment caused by a hemodynamic alteration. Although tachypnea and intercostal retractions with increased work of breathing are frequently observed in these patients, the secondary pulmonary involvement has not been well defined, either clinically or pathogenetically. Despite this, little is known $(1,2)$ about the effects of cardiac surgery on respiratory mechanics in infants with CHD nor about the time in which these changes occur. This is probably due to the lack of a simple, noninvasive method for repeatedly measuring mechanics of breathing in infants. We used the passive flow-volume single-breath technique (3) that, avoiding the necessity of esophageal pressure measurements, allowed us to perform sequential measurements with minimal disturbance to the infants.

The present longitudinal study was undertaken to investigate the respiratory and hemodynamic effects of left-to-right shunting in infants undergoing surgical repair and to investigate the sequential short-term changes after surgery, to more precisely define the course of respiratory function after correction of the hemodynamic disorder.

\section{MATERIALS AND METHODS}

We studied 31 infants ( 15 males, 16 females), aged $4 \mathrm{~d}$ to 24 mo (mean, $6.7 \mathrm{mo}$ ) who were undergoing surgical treatment for CHD with left-to-right shunts and resultant increased PBF. Body weight ranged from 2.7 to $11.8 \mathrm{~kg}$ (mean, $5 \mathrm{~kg}$ ). Characteristics and diagnoses of the subjects who completed the study are given in Table 1. Body weight was below the 3 rd percentile for age in $55 \%$ of the infants, and length was below the 3rd percentile for age in $20 \%$ of the infants. Eight infants had Down syndrome. Children were enrolled in our protocol after complete echocardiographic assessment. All children were subjected to a corrective operation, except for two infants in which palliative surgical treatment was performed (pulmonary artery banding). The protocol was approved by our Institutional Review Board; informed written consent was obtained from parents of the infants.

Echocardiographic and Doppler data allowed us to divide this 
Table 1. Characteristics of subjects*

\begin{tabular}{|c|c|c|c|c|}
\hline Case no. & Sex & Age (mo) & $\mathrm{Wt}(\mathrm{kg})$ & Diagnosis \\
\hline \multicolumn{5}{|c|}{$\begin{array}{l}\text { Group } 1 \text { (elevated PBF } \\
\text { and PAP) }\end{array}$} \\
\hline 1 & $\mathrm{M}$ & 4 & 4.9 & VSD \\
\hline 2 & M & 1.5 & 4 & CoAo, VSD \\
\hline 3 & $\mathrm{~F}$ & 0.1 & 3.5 & TGA \\
\hline 4 & M & 1 & 3.2 & ASD, VSD \\
\hline 5 & $\mathrm{~F}$ & 7 & 4.4 & VSD \\
\hline 6 & $M$ & 3.5 & 5.3 & VSD \\
\hline 7 & $\mathrm{~F}$ & 5.5 & 4.7 & CAVC \\
\hline 8 & $\mathrm{~F}$ & 4.5 & 3.6 & CAVC \\
\hline 9 & $\mathrm{~F}$ & 4.5 & 4.7 & CAVC \\
\hline 10 & M & 14.5 & 7.5 & VSD \\
\hline 11 & M & 1 & 3.2 & SV \\
\hline 12 & $M$ & 1 & 3.1 & ASD, VSD \\
\hline 13 & M & 3.5 & 4.7 & CAVC \\
\hline 14 & M & 6 & 5.6 & ASD, VSD \\
\hline 15 & $\mathrm{~F}$ & 6.5 & 5.5 & VSD \\
\hline 16 & $\mathrm{~F}$ & 3.5 & 4.2 & CAVC \\
\hline 17 & $\mathrm{~F}$ & 6 & 5.7 & CAVC \\
\hline 18 & $\mathrm{~F}$ & 0.5 & 2.7 & CAVC \\
\hline Mean & & 4.1 & 4.5 & \\
\hline \multicolumn{5}{|c|}{$\begin{array}{c}\text { Group } 2 \text { (elevated PBF } \\
\text { and normal PAP) }\end{array}$} \\
\hline 1 & M & 12 & 7 & ASD \\
\hline 2 & $\mathrm{~F}$ & 0.7 & 3.3 & ASD \\
\hline 3 & $\mathrm{~F}$ & 24 & 11.8 & VSD \\
\hline 4 & M & 13 & 5.4 & ASD \\
\hline 5 & $\mathrm{~F}$ & 4 & 4.6 & ASD \\
\hline 6 & $\mathrm{~F}$ & 4.5 & 5 & PDA \\
\hline 7 & $F$ & 8 & 6.3 & VSD \\
\hline 8 & $F$ & 17 & 11.7 & PDA \\
\hline Mean & & 10.4 & 6.9 & \\
\hline
\end{tabular}

* VSD, ventricular septal defect; CoAo, coarctation of the aorta; TGA. transposition of great arteries; ASD, atrial septal defect: CAVC, common atrioventricular channel; SV, single ventricle; and PDA, patent ductus arteriosus.

population into the following two groups: group $1(n=23)$ consisted of patients who had elevated PBF and high PAP; group $2(n=8)$ was composed of infants with elevated PBF without pulmonary artery hypertension. All children were clinically stable and breathing room air at the time of testing. Infants requiring ventilatory support were excluded. No other pulmonary diseases were present at the time of study.

Pulmonary function testing. Sequential respiratory mechanics measurements were performed, including evaluation of passive Crs and Rrs of the respiratory system. These studies were performed $24 \mathrm{~h}$ before and $10 \mathrm{~d}$ and $4-5 \mathrm{wk}$ after surgical correction.

The passive expiratory flow-volume technique (single-breath occlusion) was used to assess pulmonary mechanics by a modification of the method described in detail by LeSouef $e t$ al. (3), using a computerized infant pulmonary function device (SensorMedics 2600, Yorba Linda, CA). With this method, occlusion of the airway at end-inspiration is used to relax the respiratory muscles via the Hering-Breuer reflex (4). At endinspiration, a slide valve briefly occludes the airway, allowing alveolar and airway pressures to equilibrate. Occlusion was maintained until relaxation of the respiratory system against the valve was verified by the computer recording a plateau pressure for at least $100 \mathrm{~ms}$. The plateau was considered to be valid if pressure variation was less than $\pm 0.012 \mathrm{kPa}$ for the duration of the plateau. The occlusion was performed during sleep in sedated infants (chloralium hydrate, $80-100 \mathrm{mg} / \mathrm{kg}$ body wt, given orally). Between occlusions, infants breathed through the valve.

The valve was connected to an appropriate size face mask (Vital Signs, Totowa, NJ) that could be fitted well by an air- inflated cuff. The resistance measured for the infants was corrected for the resistance of the pneumotachograph system $(0.37$ $\mathrm{kPa} \cdot \mathrm{L}^{-1} \cdot \mathrm{s}$ ). The dead space of the system (valve-pneumotachograph) was $5.5 \mathrm{~mL}$. Infants were studied in the supine position, with the head in the neutral position and the mandible supported. The adequacy of airway occlusion also was evaluated by direct inspection of the postocclusion pressure tracing (the computer allowed for visual inspection of all breaths by graphically displaying simultaneous tracings of pressure, flow, and volume). Airway pressure was measured through a port in the valve system, connected by stiff tubing to a model DP 250-32 differential pressure transducer (linear to $\pm 9.8 \mathrm{kPa}$ ) (Validyne Engineering Corp., Northridge, CA). The valve then rapidly opened to allow passive expiration through a Hans Rudolph (Kansas City, MO) pneumotachograph (Lilly type, linear to $30 \mathrm{~L} / \mathrm{min}$ ) connected to a Validyne model DP 250-14 differential pressure transducer (linear to $\pm 0.196 \mathrm{kPa}$ ). In these relaxed expirations, the straight line of the flow-volume relationship was extrapolated to zero flow to obtain the total passive expiratory volume and to zero volume to obtain the flow appropriate for the occlusion pressure. These values were used to calculate the $\mathrm{Crs}(\mathrm{Crs}=$ total passive expiratory volume/airway occlusion pressure) and the Rrs (Rrs = airway occlusion pressure/flow).

Curves were acceptable for analysis if the pressure plateau was stable. The flow-volume curve was inspected, and a straight line to the linear expiratory portion was fitted. The first portion of the curve was usually discarded because of transients after release of the occlusion, as was the last portion of the curve that can be altered because of active expiration. By doing this, analysis of the flow-volume curve is performed between $80 \%$ and $5-10 \%$ of expired volume (5). Each measurement session was approximately 10-20 min. The pneumotachograph was calibrated with a precision air syringe using digital integration techniques $(256$ samples/s) that enabled both flow and volume to be calibrated by passing known volumes through the pneumotachograph and to be checked for linearity (128-point linearization table) before each measurement. Pressure was calibrated using a water manometer. Ten to 20 breaths were studied in each testing session; mean values were used.

To evaluate SCrs, i.e. compliance corrected for lung volumes, we measured FRC by an automated technique based on the open-circuit $\mathrm{N}_{2}$ washout (6). The technique is based on continuous analysis of the $\mathrm{N}_{2}$ concentration washed out from the lungs of a patient who breathes through a system with constant gas flow. It consists of a stable oxygen flow meter that delivers a continuous gas flow (adjusted slightly above the patient's inspiratory flow) to a tubing system. A three-way valve connects the patient to a side port, or to the washout circuit. The gas from a mixing chamber is sampled with a nitrogen detector; the data are then measured by a computer system for integration. Before the measurement starts, only pure oxygen flows through the system. The patient is connected to the system by switching the three-way valve at end-expiration. The system is calibrated by a two-point calibration method as described by Sivan et al. (7). Two volumes of room air (one above and one below the patient's estimated FRC) are injected into the circuit. The amount of $\mathrm{N}_{2}$ washed out from the infant's lung is proportional to the integrated $\mathrm{N}_{2}$ concentration. At least two FRC measurements were performed during each testing session. FRC was determined in 15 infants in group 1 and six infants in group 2.

To compare the results with those found in a reference population, compliance values were expressed as percent predicted for body length using a regression equation ( $\mathrm{Crs}=5.36 \cdot 10^{-4}$. length ${ }^{2.27}$ ) obtained from Marchal and Crance (8) on healthy infants. To compare FRC, we used the regression equation obtained from Tepper and Asdell (9) using the same $\mathrm{N}_{2}$ washout technique $(\mathrm{FRC}=$ length $\cdot 5.28-185)$.

Reproducibility. To assess the reproducibility of respiratory Crs and Rrs measurements, repeated respiratory function studies were analyzed within $30 \mathrm{~min}$ of each other in nine infants. 
Echocardiographic measurements. All patients were studied using a Vingmed CFM 700 (Horten, Norway) two-dimensional, Doppler and color-flow mechanical system with $7.5-$ or $5-\mathrm{MHz}$ phased-array annular transducers. According to our institution's policy, which does not include a routine cardiac catheterization in the preoperative assessment of CHD patients, a complete echocardiographic study was performed in every case and an additional hemodynamic study was performed only in patient 3 of group 2. The anatomical evaluation was done using parasternal, suprasternal, and subxiphoid projections. The Bernoulli simplified equation was used for the Doppler estimation of the right ventricular and pulmonary artery systolic pressures by means of the systolic pressure gradient across the ventricular septal defect or the tricuspid regurgitation (10). Direct pulmonary blood flow was not measured, but according to the literature (11) we used the Rpa/Ao size ratio as an index of the PBF status. All measurements were taken on the screen, using proper calipers, and stored on cassette and hard copy. Suprasternal or parasternal long-axis view of the right pulmonary artery and subxiphoid view of the descending aorta were carefully detected, and the transverse diameter was measured. We chose to use the diastolic dimension to avoid systolic distortions of the vessels. In the healthy child, the Rpa/Ao size ratio is 1 , and values greater than 1 are an index of increased PBF (11).

A complete echocardiographic study was performed before surgery and at the time of the respiratory parameters' controls. All measurements were performed by an operator who had no knowledge of the pulmonary function test data.

Statistical analysis. Results are reported as mean with $95 \%$ CI. Analysis of variance with subsequent modified $t$ test (Duncan) was used for analysis between time intervals. Regression analysis was used to determine whether values of Crs were correlated with values of PAP or PBF. Reproducibility was evaluated by the method of Bland and Altman (12). A $p<0.05$ was considered significant.

\section{RESULTS}

A reliable assessment of pulmonary mechanics was possible in 26 of the 31 infants initially enrolled in our protocol. Two patients were excluded because of active expiration and persistent failure to evoke Hering-Breuer reflex. Three patients belonging to the group with pulmonary hypertension died in the postoperative period. Therefore, the protocol was completed by 18 infants in group 1 and by eight infants in group 2 . The results are reported in Table 2.

Crs. Preoperative Crs values (mean with $95 \% \mathrm{CI}$ ) were 34.8 (26.5-43.1) $\mathrm{mL} \cdot \mathrm{kPa}^{-1}$ for group 1 and 95 (53.1-136.9) $\mathrm{mL}$. $\mathrm{kPa}^{-1}$ for group 2. The first postoperative control (Post 1) showed a significant $(p<0.01)$ improvement of Crs values in group 1 , whereas group 2 values remained statistically unchanged (Table 2 ). When expressed as percent of predicted value (8), group 1 presented a mean Crs value of $60 \%$ before surgery that improved to $82 \%$ and $98 \%$ in the two postsurgery sessions. Preoperative Crs values (normalized by length) were significantly lower $(p<$ 0.01 ) in group 1 with respect to group 2. When expressed as percent of predicted value, group 2 presented a mean Crs value of $113 \%$ before surgery and of $105 \%$ and $118 \%$ in the two postoperative sessions.

The $\mathrm{CV}$ for compliance (mean with $95 \% \mathrm{CI}$ ) of the three sessions study was $8.6 \%(7.1-10.1 \%)$. Individual values are shown in Table 3.

SCrs. When compliance was related to lung volume (i.e. specific compliance), we noted a trend similar to that for Crs with a significant $(p<0.01)$ postoperative improvement in group 1 and no significant changes ( $p=$ NS) in group 2 (Table 2).

Rrs. In both group 1 and group 2, Rrs preoperative values ranged within normal limits. No significant changes were found in mean Rrs values in either group 1 or group 2 after surgical correction of the left-to-right shunt (Table 2). The CV for resistance was $10.9 \%(8.8-13 \%)$. Individual values are shown in Table 3.

$F R C$. FRC values are shown in Table 2. When expressed as percent of predicted value (9), we found, before surgery, a mean value of $101 \%$ and $112 \%$ in group 1 and 2, respectively. No significant changes occurred after surgical treatment in either group.

Rpa/Ao ratio. $\mathrm{Rpa} /$ Ao ratio in the total population (mean with $95 \% \mathrm{CI}$ ) was $1.36(1.20-1.52)$ before surgery, and 1.09 $(1.01-1.17)$ and $1.03(0.84-1.22)$ at the first and second postoperative controls, respectively. These values differed significantly among themselves $(p<0.05)$. No significant correlation was found between $\mathrm{Rpa} /$ Ao size ratio and values of $\mathrm{Crs}(r=0.4, p=$ NS).

$P A P$. Before surgery, PAP values (mean with $95 \% \mathrm{CI}$ ) were $9.75(8.97-10.53)$ and $3.96(3.27-4.65) \mathrm{kPa}$ in group 1 and group 2 , respectively. After surgical correction. PAP values fell to 4.83 $(4.58-5.08) \mathrm{kPa}$ in group $1(p<0.001)$, whereas no significant changes $(p=N S)$ occurred in group 2 , with a value of 3.48 $(3.19-3.77) \mathrm{kPa}$. No significant correlation was found between PAP and Crs values ( $r=0.4, p=\mathrm{NS})$.

Considering the eight Down syndrome patients as a separate subgroup, no significant differences were found between their values of PAP and Rpa/Ao ratio and those of the other group 1 children.

Reproducibility: Paired observations for Crs and Rrs with repeated measurements in nine infants showed good correlation coefficients (Crs: $r=0.99$, slope $=0.97$; Rrs: $r=0.97$, slope $=$ 0.91 ). The coefficient of repeatability was calculated for Crs and $\mathrm{Rrs}$ as reported by Bland and Altman (12) and resulted in values of $4.32 \mathrm{~mL} \cdot \mathrm{kPa}^{-1}$ for Crs and $0.44 \mathrm{kPa} \cdot \mathrm{L}^{-1} \cdot \mathrm{s}$ for Rrs. All differences between the first and the second measurement were within these limits, for both Crs and Rrs.

Table 2. Respiratory function data*

\begin{tabular}{|c|c|c|c|c|}
\hline & & PRE & POST 1 & POST 2 \\
\hline \multicolumn{5}{|l|}{ Group ! $(n=18)$} \\
\hline $\operatorname{Crs}\left(\mathrm{mL} \cdot \mathrm{kPa}^{-1}\right)$ & 34.8 & $(26.5-43.1)$ & $47.5 \dagger(39.2-55.8)$ & $56.5+(45.6-67.4)$ \\
\hline $\operatorname{Rrs}\left(\mathrm{kPa} \cdot \mathrm{L}^{-1} \cdot \mathrm{s}\right)$ & 5.6 & $(4.09-7.15)$ & $5.71 \mp(4.69-6.73)$ & $5.35 \ddagger(4.45-6.25)$ \\
\hline $\operatorname{SCrs}\left(\mathrm{kPa}^{-1}\right)$ & 0.2 & $(0.24-0.30)$ & $0.44 \dagger(0.42-0.46)$ & $0.44 \dagger(0.41-0.47)$ \\
\hline $\mathrm{FRC}(\mathrm{mL})$ & 130 & $(100-160)$ & $114 \ddagger \quad(94-134)$ & $124 \ddagger \quad(99-149)$ \\
\hline \multicolumn{5}{|l|}{ Group $2(n=8)$} \\
\hline $\operatorname{Crs}\left(\mathrm{mL} \cdot \mathrm{kPa}^{-1}\right)$ & 95 & $(53.1-136.9)$ & $81.7 \ddagger(50.1-113.3)$ & $111.2 \ddagger(62-160.4)$ \\
\hline $\operatorname{Rrs}\left(\mathrm{kPa} \cdot \mathrm{L}^{-1} \cdot \mathrm{s}\right)$ & 4.0 & $(3.32-4.8)$ & $3.84 \ddagger(2.9-4.78)$ & $4.0 \ddagger(2.92-5.08)$ \\
\hline $\operatorname{SCrs}\left(\mathrm{kPa}^{-1}\right)$ & 0.5 & $(0.48-0.56)$ & $0.45 \ddagger(0.42-0.48)$ & $0.58 \ddagger(0.52-0.64)$ \\
\hline $\mathrm{FRC}(\mathrm{mL})$ & 205 & $(140-270)$ & $219 \ddagger \quad(132-306)$ & $206 \ddagger \quad(102-310)$ \\
\hline
\end{tabular}

* Values are means 95\% CI. Data are before (PRE), $10 \mathrm{~d}$ after (POST 1), and 4-5 wk after (POST 2) cardiac surgery. FRC measurement was determined in 15 infants of group 1 and in six children of group 2.

$\dagger p<0.01$ from the presurgery value.

$\ddagger p=\mathrm{NS}$. 
Table 3. Individual values of Crs, Rrs, and respective $\mathrm{CV}^{*}$

\begin{tabular}{|c|c|c|c|c|c|c|c|c|}
\hline & \multicolumn{4}{|c|}{ PRE } & \multicolumn{4}{|c|}{ POST 2} \\
\hline & $\begin{array}{c}\text { Crs } \\
\left(\mathrm{mL} \cdot \mathrm{kPa}^{-1}\right)\end{array}$ & $\mathrm{CV}$ & $\begin{array}{c}\mathrm{Rrs} \\
\left(\mathrm{kPa} \cdot \mathrm{L}^{-1} \cdot \mathrm{s}\right)\end{array}$ & $\mathrm{CV}$ & $\begin{array}{c}\text { Crs } \\
\left(\mathrm{mL} \cdot \mathrm{kPa} \mathrm{a}^{-1}\right)\end{array}$ & $\mathrm{CV}$ & $\begin{array}{c}\operatorname{Rrs} \\
\left(\mathrm{kPa} \cdot \mathrm{L}^{-1} \cdot \mathrm{s}\right)\end{array}$ & $\mathrm{CV}$ \\
\hline \multicolumn{9}{|c|}{ Group 1} \\
\hline 1 & 18.2 & 9.9 & 4.12 & 9.9 & 56.9 & 4.4 & 4.21 & 9 \\
\hline 2 & 24.1 & 9.4 & 6.57 & 6.1 & 57.5 & 8.6 & 6.17 & 4.9 \\
\hline 3 & 15.7 & 18 & 5.88 & 30.9 & 27.8 & 6.8 & 6.37 & 12.8 \\
\hline 4 & 12.7 & 9.9 & 1.76 & 7.6 & 44.3 & 7.6 & 9.11 & 6.5 \\
\hline 5 & 20.7 & 9 & 5.19 & 4.9 & 25.9 & 7.6 & 3.23 & 10.6 \\
\hline 6 & 65.1 & 4 & 3.43 & 7.1 & 65.8 & 11.2 & 4.41 & 10.4 \\
\hline 7 & 61.3 & 4.5 & 6.96 & 9.2 & 83.9 & 5.7 & 10.19 & 5.5 \\
\hline 8 & 45.7 & 7.5 & 3.33 & 9 & 50.6 & 9.5 & 4.70 & 9.9 \\
\hline 9 & 27.8 & 9.5 & 1.96 & 13.2 & 75.7 & 10 & 3.72 & 13.7 \\
\hline 10 & 58.1 & 10.1 & 1.86 & 8.3 & 111 & 5.3 & 2.84 & 12.9 \\
\hline 11 & 16.7 & 8.4 & 7.64 & 8.7 & 26.5 & 6.9 & 4.99 & 14.9 \\
\hline 12 & 19.8 & 11.3 & 4.31 & 9.2 & 23.3 & 8.8 & 5.49 & 7.5 \\
\hline 13 & 31.6 & 8.6 & 4.12 & 5.6 & 68.8 & 5.4 & 6.66 & 4.5 \\
\hline 14 & 67.6 & 5.3 & 5.10 & 12 & & & & \\
\hline 15 & 36.9 & 6.7 & 5.19 & 14.7 & 68.4 & 10.2 & 5.49 & 11.5 \\
\hline 16 & 33.4 & 7.7 & 5.68 & 11 & 56.3 & 10.7 & 4.41 & 7.8 \\
\hline 17 & 50.7 & 6.3 & 6.17 & 6.4 & 73.9 & 4.5 & 5.49 & 7.6 \\
\hline 18 & 19.9 & 10.6 & 5.98 & 11.8 & 43.8 & 11.1 & 3.43 & 12.7 \\
\hline \multicolumn{9}{|c|}{ Group 2} \\
\hline 1 & 56.4 & 8.7 & 3.92 & 7.3 & 87 & 7.6 & 4.31 & 14.6 \\
\hline 2 & 44.3 & 8.4 & 4.61 & 9.9 & 40.6 & 7.9 & 5.98 & 6.5 \\
\hline 3 & 223.1 & 6.5 & 2.45 & 8.3 & 224.5 & 10 & 4.12 & 9.6 \\
\hline 4 & 109.1 & 9.6 & 4.21 & 15.2 & & & & \\
\hline 5 & 52.1 & 9.9 & 4.41 & 12.9 & 77 & 10.3 & 3.33 & 10.9 \\
\hline 6 & 29.1 & 11 & 6.08 & 13.9 & 80.9 & 5.0 & 3.92 & 10.8 \\
\hline 7 & 107 & 10.8 & 4.12 & 12.9 & & & & \\
\hline 8 & 145.2 & 9.2 & 2.65 & 9.7 & 157.2 & 7.6 & 2.25 & 7.7 \\
\hline
\end{tabular}

* Values are before (PRE) and 4-5 wk after (POST 2) cardiac surgery.

\section{DISCUSSION}

It is well known that abnormal pulmonary hemodynamics can cause a derangement of the mechanical properties of the lung, often producing clinical disturbances of respiration with subsequent increased work and oxygen cost of breathing $(1,2,13)$. The findings of the present study show that the surgical treatment of CHD with left-to-right shunting promptly improves respiratory mechanics in infants who present an increase in both PAP and PBF (group 1). In this group of infants, as soon as $10 \mathrm{~d}$ after surgical correction, we found a mean $50 \%$ significant improvement in compliance. By 4-5 wk postoperatively, compliance reached values that can be considered within the normal range for healthy infants (8).

Most of the previous research in children with left-to-right shunts are based on a single preoperative assessment of lung mechanics $(13,14)$. The marked differences in the patient populations studied and in the measurement technique used make the comparison of these reports difficult. Despite this, it is widely known that infants with large left-to-right shunting and elevated PAP have decreased values of pulmonary compliance; whereas in subjects without associated pulmonary artery hypertension, slight or no deterioration of respiratory mechanics is described $(1,2,13)$. Bancalari et al. (13), studying infants with increased and decreased PBF, suggested that PAP, and not PBF, is the primary factor that affects compliance in patients with left-toright shunts. The preoperative results of the present study are consistent with this conclusion. In fact, before surgical correction, the group with normal PAP (group 2) presented mean Crs values that can be considered within the normal range $(113 \%$ of predicted value) reported in the literature for passive compliance (8). Conversely, group 1 presented a mean compliance value of $60 \%$ with respect to predicted values (8).

The lack of precise agreement on predicted values in this age group, however, makes it difficult to compare our patients with healthy children, primarily because we are dealing with a population of growth-retarded infants. This is an important factor that needs consideration when interpreting compliance and FRC values from this study using prediction equations for weight or length. Of the published data available evaluating passive compliance in healthy infants, most agree with the data of Marchal and Crance (8) using the multiple occlusion technique. However, the reference values reported are not definitive because no value is based on a large population of subjects studied. For this reason, it is recognized that the most complete value of pulmonary function testing at this age consists of a longitudinal evaluation of the patients, for both clinical aims and experimental works, with each patient serving as his or her own control (14). To our knowledge, little information is available about the results of serial pulmonary function assessment in CHD infants studied before and after surgical treatment $(1,2)$. As in our report, Griffin et al. (2) found low preoperative Crs values with a marked improvement several weeks after surgical correction in a group of infants with CHD. However, they did not provide a statistical analysis of their data, precise information with respect to the age of the children studied, or the time of pulmonary function assessment in relation to the surgical correction. In a large study of pulmonary mechanics in normal and CHD children, Howlett (1) performed serial pulmonary function assessment in only five infants with left-to-right shunts, reporting a significant improvement in compliance values within a few days after the surgical treatment. To our knowledge, this is the only work presenting results obtained in the immediate preoperative and postoperative periods.

Unlike Bancalari et al. (13), we found no correlation between PAP and Crs values. This observation, in agreement with the data of Howlett (1), could indicate that a coupling of both high $\mathrm{PAP}$ and PBF is responsible for the reduced lung elastic proper- 
ties in children with significant left-to-right shunt. There is limited information about the relationship between PAP and lung compliance both in animal models and humans. In animal models, some investigators failed to detect any changes in respiratory function after experimental changes in PAP $(15,16)$. On the other hand, other investigators found a clear correlation between PAP and lung compliance (17). The time course and the severity of the events seem to depend not only on the size of the shunt but also on individual factors, type of abnormality, and duration of the altered pulmonary hemodynamic and subsequent pulmonary vascular remodeling $(18,19)$. This may explain the variability with regard to the relationship between lung compliance and PAP.

Another alteration described in children with CHD is an elevation in pulmonary resistance (13). In our study, resistance was within the normal range reported in the literature $(4,20)$ in both groups of infants. It persisted unchanged even when the hemodynamic defects were surgically corrected.

Although it is known that measurements of compliance can be difficult to interpret when FRC is not being measured, only a few studies $(1,13)$ have reported data with regard to specific compliance (i.e. compliance corrected for lung volumes) in infants with $\mathrm{CHD}$. The evidence that no significant differences in FRC values were observed between preoperative and postoperative measurements suggests that the improvement in compliance was related to changes in the elastic properties of the lung and not to differences in lung volume. This latter observation is consistent with the study of Howlett (1), in which FRC was measured in five infants with left-to-right shunts before and after surgery. Because of the discrepancy between lung volumes measured using plethysmographic versus gas dilution technique, comparison of our FRC values with those obtained by Howlett (1) in CHD infants is not possible. The FRC results obtained in the present study using an open-circuit $\mathrm{N}_{2}$ washout technique are comparable to the values recently reported by Tepper and Asdell (9) in healthy infants when FRC was normalized for body length. They also demonstrated that helium dilution and nitrogen washout techniques for measuring FRC yield accurate and comparable results.

Most data available in the literature about respiratory function in infants with CHD are based on esophageal pressure measurements for calculating dynamic lung compliance $(1,2,13)$. This invasive method might be considered unreliable in infants with chest wall retraction because of regional inequalities in pleural pressure $(21,22)$. We used the passive flow-volume technique; this is a noninvasive measurement of respiratory mechanics validated in animal studies $(23,24)$ and in healthy and sick infants $(3,4,25-27)$. Recently, Davies et al. (14) demonstrated the applicability of the occlusion technique in infants with CHD. This technique is based on the relaxation of respiratory muscles after the occlusion of the airways because of Hering-Breuer reflex. This lung inflation reflex seems to persist throughout the first two years of life $(28,29)$, and, recently, Greenough and Pool (30) have found this reflex can be evoked in asthmatic children aged 5-7 y. The reliability of measurements in our study can be considered acceptable with no difference in Crs values in repeated measurements obtained within $30 \mathrm{~min}$, consistent with the results of Davies et al. (14) using the multiple occlusion technique.

Potential problems of the single-breath occlusion technique regard the assumption that the respiratory system can be treated as a single compartment. This may not be true in very sick infants, especially in presence of severe bronchoconstriction in which a two-compartment model may be present (5). Other issues that need to be investigated using the occlusion technique regard the possible influences of glottic narrowing in modulating expiratory flow.

In conclusion, the results of this study show that infants with left-to-right shunts and pulmonary hypertension present a reduced compliance of the respiratory system with normal resistance, and that surgical repair of the hemodynamic disorder is followed by a rapid and significant improvement of respiratory function.

\section{REFERENCES}

1. Howlett $G 1972$ Lung mechanics in normal infants and infants with congenital heart disease. Arch Dis Child 47:707-715

2. Griffin AJ. Ferrara JD, Lax JO, Cassels DE 1972 Pulmonary compliance. An index of cardiovascular status in infancy. Am J Dis Child 123:89-95

3. LeSouef PN, England SJ, Bryan AC 1984 Passive respiratory mechanics in newborn and children. Am Rev Respir Dis 129:552-556

4. Mortola J, Fisher J, Smith B, Fox G. Weeks S 1982 Dy namics of breathing in infunts. J Appl Physiol 52:1209-1215

5. Dezateaux CA. Fletcher ME, Rabbette PS. Stanger LJ. Stocks J 1991 A Manual of Infant Lung Function Testing. Portex Anaesthesia, Intensive Therapy and Respiratory Medicine Unit, Institute of Child Health. London, p 15!

6. Gerhardt T, Reifenberg L. Hehre D. Feller L. Bancalari E 1986 Functional residual capacity in normal neonates and children up to 5 years of age determined by a $\mathrm{N}_{2}$ washout method. Pediatr Res 20:668-671

7. Sivan Y, Deakers TW, Newth CJ 1990 An automated bedside method for measuring functional residual capacity by $\mathrm{N}_{2}$ washout in mechanically ventilated children. Pediatr Res 28:446-450

8. Marchal F, Crance JP 1987 Measurement of ventilatory system compliance in infants and young children. Respir Physiol 68:311-318

9. Tepper RS, Asdell S 1992 Comparison of helium dilution and nitrogen washout measurements of functional residual capacity in infants and very young children. Pediatr Pulmonol 13:250-254

10. Williams RG. Bierman FZ. Sanders SP 1986 Echocardiographic Diagnosis of Cardiac Malformations. Little, Brown \& Co. Boston, p 227

11. Snider AR, Enderlein MA. Teitel DF, Juster RP 1984 Two-dimensional echocardiographic determination of aortic and pulmonary artery sizes from infancy to adulthood in normal subjects. Am J Cardiol 53:218-224

12. Bland JM. Altman DG 1986 Statistical methods for assessing agreement between two methods of clinical measurement Lancet 1:307-310

13. Bancalari E, Jesse MJ, Gelband H, Garcia O 1977 Lung mechanics in congenital heart disease with increased and decreased pulmonary blood flow. J Pediatr 90:192-195

14. Davies CJ, Cooper SG, Fletcher ME, Hatch DJ, Helms PJ, Gordon 1, Stocks J 1990 Total respiratory compliance in infants and young children with congenital heart disease. Pediatr Pulmonol 8:155-161

15. Caeton AJ, Goetzman BW, Bennet SH. Milstein JM 1987 Effect of pulmonary hypertension on lung compliance in newborn lamb. Pediatr Pulmonol 3:324-327

16. Perez Fontan JJ, Clyman RI, Mauray F, Heymann MA, Roman C 1987 Respiratory effects of a patent ductus arteriosus in premature newborn lambs. J Appl Physiol 63:2315-2324

17. Rendas A, Lennox S, Reid L 1979 Aorta-pulmonary shunts in growing pigs. J Thorac Cardiovasc Surg 77:109-118

18. Rabinovitch M 1988 Problems of pulmonary hypertension in children with congenital cardiac defects. Chest 93:119S-126S

19. Stenmark KR. Orton EC, Reeves JT, Voelkel NF, Crouch EC, Parks WC. Mecham RP 1988 Vascular remodeling in neonatal pulmonary hypertension. Chest 93:127S-133S

20. Marchal F, Haouzi P, Gallina C. Crance JP 1988 Measurement of ventilatory system resistance in infants and young children. Respir Physiol 73:201-210

21. LeSocuf PN, Lopes JM, England SJ, Bryan MH. Bryan AC 1983 Influence of chest wall distortion on esophageal pressure. J Appl Physiol 55:353-358

22. Heaf DP, Turner H, Stocks J, Helms P 1986 The accuracy of esophageal pressure measurements in convalescent and sick infants. Pediatr Pulmonol
$2: 5-8$

23. Davis GM, Coates AL, Dalle D, Bureau M 1988 Measurements of pulmonary mechanics in the newborn lamb: a comparison of three techniques. J Appl Physiol 64:972-981

24. Cunningham J, Morgan W, Lemen R, Witten M, Magarelli J, Quan S 1987 Passive exhalation technique correlates with esophageal balloon measurements of respiratory mechanics in beagle nups. Am Rev Respir Dis 136:722ments

25. Lodrup KC. Mowinckel P. Carlsen KH 1992 Lung function measurements in awake compared to sleeping newborn infants. Pediatr Pulmonol 12:99-104

26. Brundage KL, Mohsini KG. Froese AB. Fisher JT 1990 Bronchodilator response to ipratropium bromide in infants with bronchopulmonary dysplasia. Am Rev Respir Dis 142:1137-1142

27. Stocks J, Nothen U, Sutherland P, Helms P 1987 Improved accuracy of the occlusion technique for assessing total respiratory compliance in infants. Pediatr Pulmonol 3:71-77

28. Rabette PS, Dezateaux CA, Fletcher ME 1991 The Hering-Breuer reflex declines during the first year of life. Eur Respir J 4:1226(abstr)

29. Witte MK, Carlo WM 1987 Prolongation of inspiration during lower airway occlusion in children. J Appl Physiol 62:1860-1864

30. Greenough A, Pool J 1991 Hering-Breuer reflex in young asthmatic children. Pediatr Pulmonol 11:345-349 